\title{
Stimulating Deep Learning Network on Graphical Processing Unit To Predict Water Level
}

\author{
Neeru Singh, Supriya P. Panda
}

\begin{abstract}
Deep learning is widespread over different fields like health industries, voice recognition, image \& video classification, real-time rendering applications, face recognition and many other domains too. Fundamentally Deep Learning is used due to the three different aspects. The first one is its ability to perform better with a huge amount of data for training, second is high computational speed, and third is the elevation of deep training at various levels of reflection and depiction.
\end{abstract}

Acceleration of Deep Machine Learning requires a platform for immense performance; this needs accelerated hardware for training convoluted deep learning problems. While training large datasets on deep learning that takes hours, days, or weeks, accelerated hardware that decreased the overload of computation task can be used. The main attention of all the research studies is to optimize the results of predictions in terms of accuracy, error rate and execution time. Graphical Processing Unit (GPU) is one of the accelerated hardware that has currently prevailed to decrease the training time due to its parallel architecture.

In this research paper, the multi-level or Deep Learning approach is simulated over Central Processing Unit (CPU) and GPU. Different research claims that GPUs deliver accurate results with a maximum rate of speed. MATLAB is the framework used in this work to train the Deep Learning network for predicting Ground Water Level using a dataset of three different parameters Temperature, Rainfall, and Water requirement. Thirteen year's dataset of Faridabad District of Haryana from the year 2006 to 2018 is used to train, verify, test and analyzed the network over CPU and GPU. The training function used was the trailm for training the network over CPU and trainscg for GPU training as it does not support Jacobian training.

From our results, it is concluded that for large dataset the accuracy of training increased with GPU and processing time for training is decreased when compared to CPU. Overall performance improves while training the network over GPU and suits to be a better method for predicting the Water Level. The proficiency estimation of the network shows the maximum regression value, least Mean Square Error (MSE), and highperformance value for GPU during the training.

Index Terms-Deep Learning, Graphical Processing Unit (GPU), Central Processing Unit, Prediction, Artificial Neural Network.

\section{INTRODUCTION}

Deep Learning that stimulates like human brain for acquiring knowledge is the subsection of Machine

Revised Manuscript Received on April 12, 2020.

* Correspondence Author

Neeru Singh*, Computer science \& Engineering department, Manav Rachna International Institute of Research and Studies, Faridabad, India. Email: neeruksingh123@gmail.com

Dr. Supriya P Panda, Computer science \& Engineering department, Manav Rachna International Institute of Research and Studies, Faridabad, India. Email: supriya.fet@mriu.edu.in

(C) The Authors. Published by Blue Eyes Intelligence Engineering and Sciences Publication (BEIESP). This is an open access article under the CC BY-NC-ND license (http://creativecommons.org/licenses/by-nc-nd/4.0/)
Intelligence. Machine Learning is the branch of Artificial Intelligence that has the ability to learn automatically from past experience without being programmed specially. Deep learning is favourable to the scientists and researchers that need to collect, analyse, and interpret large amount of data. Deep Learning is used to automate the predictive analysis. Traditional Machine learning approach for training network are linear and that of Deep learning is hierarchical with layered architecture.

In Machine learning an optimum criteria heading to accelerate the accurate categorization and need escalation to increase the performance by continuous training until the error is reduced [1].

Artificial Neural Network and Deep Learning became the most used architecture to train the network due to the advancement in hardware like Graphical Processing Unit (GPU). Due to the performance improvement of GPU with low cost, it is highly used for deep training large data sets. Using GPUs for training deep networks gives accurate results within less time as compared to Central Processing Unit (CPU). Deep learning training works with a large number of very time-consuming matrix operations. To overcome this issue GPUs are used to speed up the deep training.

As shown in "fig.1" Deep Learning is the subclass of Neural Network, which is the part of Machine Learning and Machine learning itself is a subclass of Artificial Intelligence [2].

Two different platforms can be used while training deep networks. One is a CPU that works both in a sequential manner and a parallel manner with multicore CPUs. While GPUs work inherently parallel with thousands of cores that act as processing units. Both the CPU and GPU work on different principles. The Central Processing Unit (CPU) executes and controls all applications similar to human brain [3].

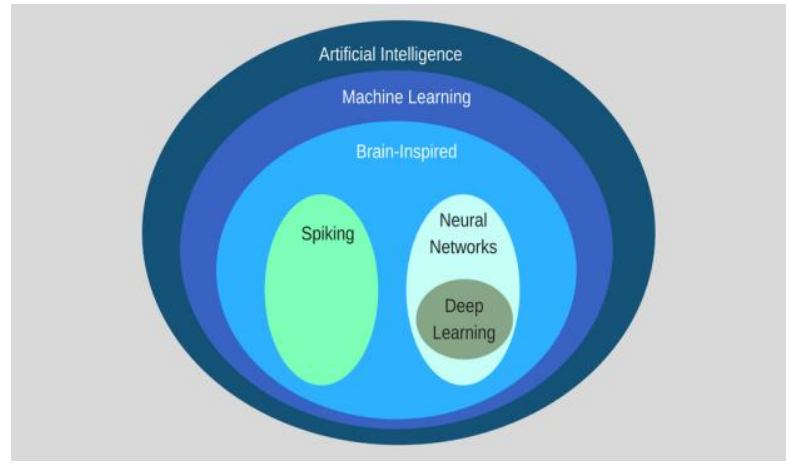

Fig. 1. AI vs. Machine Learning vs. Deep Learning [2] 
CPU is designed for improving performance by simply executing one single task at one time to implement fast response time. On the other hand, GPUs are designed to work with graphics like rendering, gaming, and other graphics functions that have large data set and a higher degree of parallelism is required. GPUs are unified on the CPU chips, and work together with CPU to praise the work of it [3]. For traditional work assignments like singlethreaded systems, CPUs are the most used platform. The number of cores that is the processing unit is very limited in CPUs so there is very less amount of parallel processing that takes place in it. GPUs have thousands of cores that mean many small processing units that execute tasks parallel [4].

This research work focuses on taking advantage of GPU environment to run massive parallel architecture for Deep Learning on low-end computers to give best approach that suits for Ground Water Level Prediction. The performance is evaluated on the basis of speed and time by comparing the results of both CPU and GPU execution.

This paper is systematized in seven different sections. Section-2 illustrates the background in the fields of Deep Learning with GPU. Section-3 describes the study area. Section-4 defines the proposed methodology used in this research. Section-5 gives detailed information about the hardware platform used in it. Section-6 demonstrates the results and findings. Section-7 presents the conclusion part and Section- 8 defines the work to be done in the future.

\section{BACKGROUND}

\section{A. Deep Learning Network (DLN)}

According to recent analysis in time series research and development Deep Learning Networks exhibits tremendous work for prediction on large dataset with more accuracy and throughput [5]. The successful applications of Deep Neural Network (DNN) have substantiate in many domains and is the most appropriate approach for performance improvement over prediction problems [6]. Given below "fig. 2 " represents the simple architecture of Deep Learning Networks where input neurons are connected to hidden neurons and successively to output layer neurons [7].

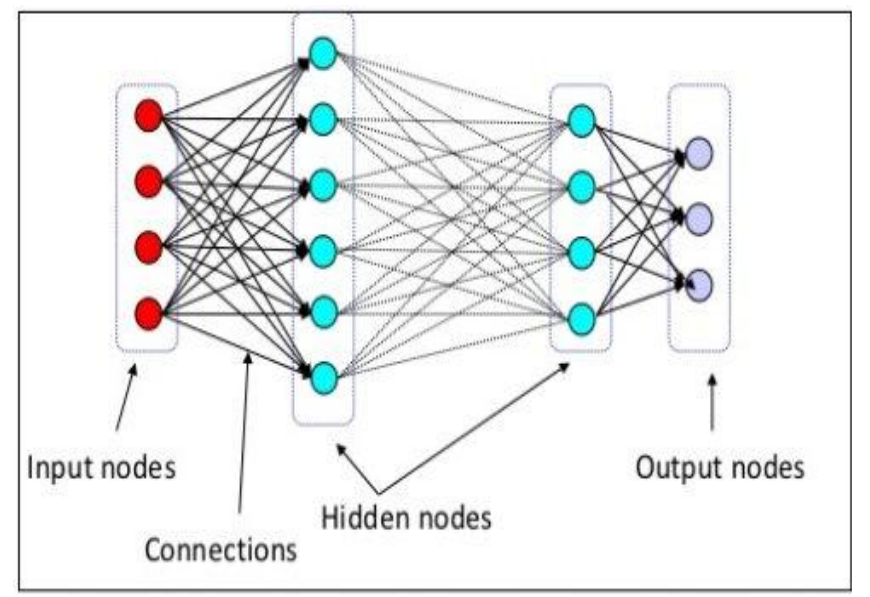

Fig. 2. Deep Learning Network [7]
An extent amount of analysis has been done for estimating the future, but the smart model that will predict the future more accurately is ANN that stands out to be the one [8].

Here the paper directs attention on the Ground Water level of Faridabad that ranges from 30 meters to 50 meters below the ground level. As the requirement of water increases constantly, the level of the groundwater continually dips down to 0.50 meters per annum from the past 30 years. The basic element of reduction of water in Haryana is due to the crops, which require more water than usual [9].

The model Artificial Neural Networks (ANNs) has been advantageous for different engineering applications including prediction and forecasting problems. By using ANN model hydrological conditions of different areas have been predicted like a prediction of water level for Rockport (Texas), Sarawak (Malaysia), Gulf of Mexico and many more [10], [11], [12].

The approach of ANN in conjoins with Multiple-Input Single Output provided the best representation of rainfall and runoff relationship and also represents the best results with accuracy on the prediction of the Narmada River [13]. Also, the prediction for flow and level of water has been done using Deep learning approach on Shannon River in Ireland [14].

ANN has been applied to estimate the groundwater level of Hatay, Amik Plain, and Kumlu district of Turkey by taking monthly mean precipitation and temperature as input, and evaluates the performance of Multi-Linear Regression (MLR) method [15].

While predicting the groundwater level of the Abhanpur block of Raipur District Chhattisgarh state in India using ANN Model ,the ANN model results in the best fit curve with a high correlation coefficient(R) [16].

According to the literature survey, one of the best models suited for the prediction is ANN, which provides more accurate results than other different approaches available for prediction.

Deep Learning is also an Artificial Neural Network (ANN) System with more depth that means it is more complex architecture. The associated complexity is embellished by the design of how the instruction flows all over the model. The working of both models is the same except the architecture that is more complex for Deep Learning.

\section{B. Graphical Processing Unit (GPU)}

As the name signifies the Graphical Processing Unit is the unit that supports graphics operations. When first they were introduced in the 1990s were proficient enough to do graphics work only. But from 2004 GPU's were more capable of doing other graphic computing. A GPU is the unit that is integrated into the CPU. GPU works with CPU to compliment it. "Fig.3" presents an image for GPU execution, which communicates with the CPU to perform complex task.

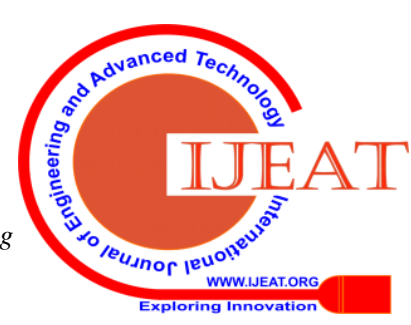




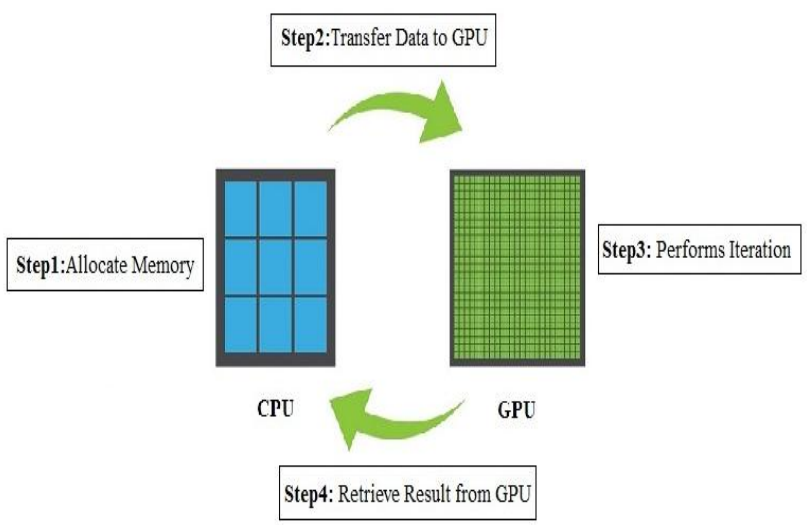

Fig. 3. GPU Execution

GPU executes the function which requires high intense computation in a parallel way, while the rest of the part is executed on CPU in a sequential manner. First data is allocated to the main memory for its execution and then a function that requires more calculations will be transferred to the GPU. Launch GPU kernels to process data and perform iteration by parallel executing in each GPU core. In the last step, the result is retrieved back to the main memory from the GPU. An approach to the multithreaded system was designed to validate the performance evaluation of the metaheuristic time series forecasting model. In this work, GPU Deep Learning training is applied to increase the total performance of the system by assigning work to the multicore processing units [17].

Earlier work proclaims that GPU is an efficient parallelization method to accelerate the Recurrent Neural Network training for online handwritten recognition by improving training speed and overall performance [18].

\section{STUDY AREA}

Faridabad District is about 32 kilometres apart from Delhi India at a distance of 3.5 kilometres from National Highway of Delhi-Agra, at the latitude of $28^{0} 25^{\prime}$ North and longitude of $77^{\circ} 18^{\prime}$ East [18]. The map of Faridabad zone is shown below in "fig.4". For better and accurate prediction results, thirteen years have been taken from 2006 to 2018. The model used in this paper is the Deep Learning approach with GPU, which gives more accurate results than Deep Learning with CPU alone.

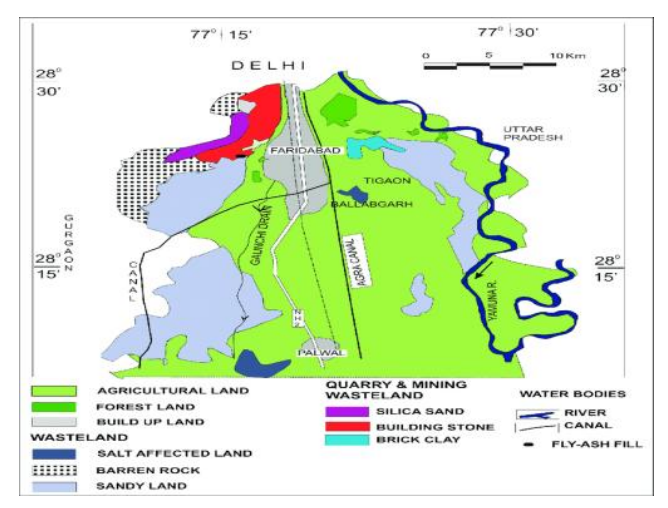

Figure 4.Faridabad Map

\section{METHODOLOGY FOLLOWED}

The methodology followed in this paper is shown below in "fig. 5 " by the flow diagram with different activities taken in stepwise function. For predicting the future value of water level, the input parameters identified were water requirement, temperature, and rainfall.

\section{A. Data Used}

Rainfall, temperature, water requirement of different years was used for Faridabad zone to predict the level of water. The data used was of thirteen years from 2006 to 2018 and collected from different sites like Water Resources Information System of India (India-WRIS), meteorological sites and different years records from Central Ground Water Board (CGWB) of Haryana. In this paper the data used for the training phase was $70 \%$ of overall data. After the training period rest 30\% data were used for validation and testing, which, means $15 \%$ for both the operation. Our hypothesis states that there is significant increase in the performance of training the data on parallel mode and GPU with that of CPU alone.

\section{B. Normalization of Data}

Before training, the data should be normalized between the specified ranges for input and output because they accept distinct values and helps in retaining the relationships with original data [20]. The transfer function used in this paper was sigmoid function and for this function, data must lie between 0 and 1 .

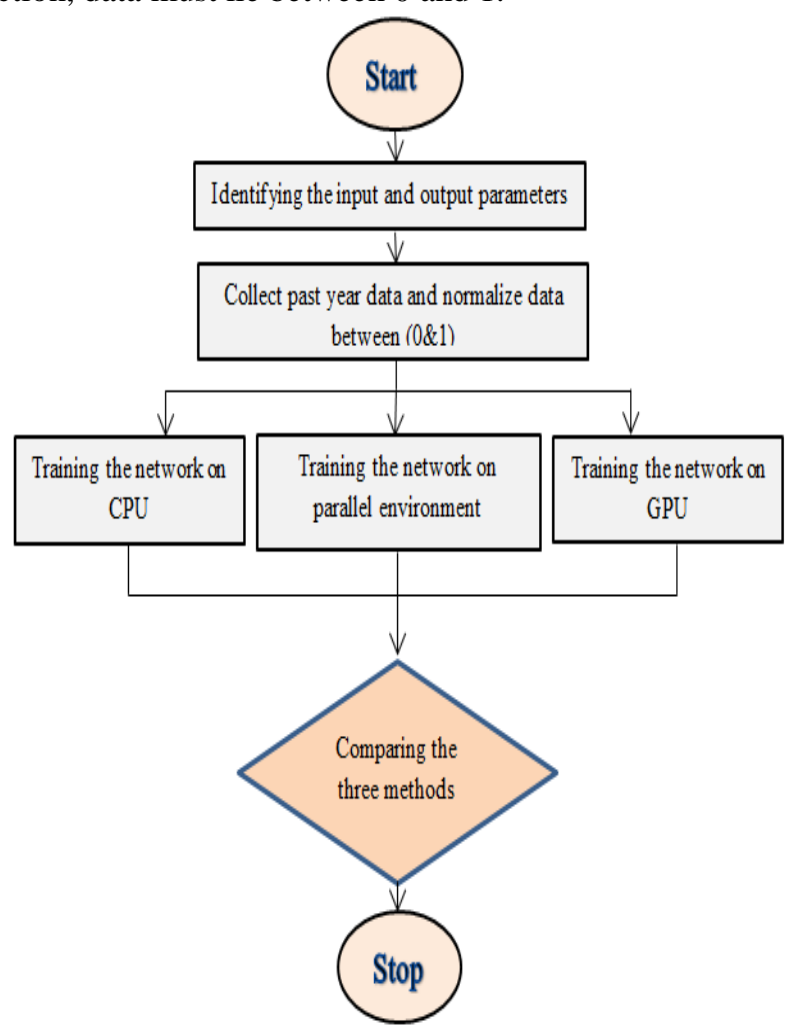

\section{Figure 5.Methodology Followed}

The formula used for normalizing the data is given below in the "(1)".

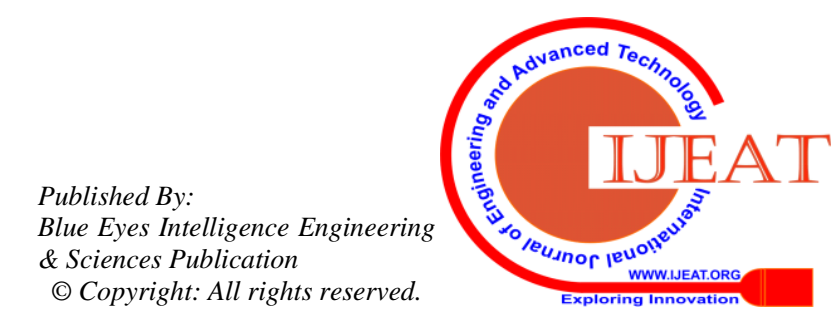


$\operatorname{Normalized}\left(x_{i}\right)=\frac{x_{i}-x_{\min }}{x_{\max }-x_{\min }}$.

, where

$\mathrm{i}=$ Value of iteration used.

$\mathrm{X}_{\min }=$ Minimum value for the data set variable $\mathrm{X}$.

$\mathrm{X}_{\max }=$ Maximum value for the data set variable $\mathrm{X}$.

\section{Architecture Used}

Deep Learning architecture has been used, along NonAutoregressive with external input (NARX) method. Its purpose is to take past values and predicted the outcome value from the same or different series. The logic behind selecting the NARX model is the ability to handle difficult situations and avoid repeated tests, like trial and error method, which minimizes the time for execution [21]. There is need to choose the number of hidden nodes and tapped delay to elect NARX model network architecture, with simulating and experimenting will find out the optimized composition of the network [22]. This is represented by the “(2)" given below:

$y(t)=f(y(t-1), . ., y(t-d), x(t-1), . .,(t-d)$.

, where

$y(t)=$ Series for predicting future value.

$\mathrm{y}(\mathrm{t}-1)=$ Past value for $\mathrm{y}$ series

$\mathrm{x}(\mathrm{t}-1)=$ Past value for second series.

"Fig.6. (a)" shows the series-parallel architecture in which the actual output is feedback and act as classic feedforward architecture with fixed backprapogation and "(b)" shows the parallel architecture in which the estimated output is feedback instead of actual output. The implementation of the network is focused on multiple inputs and one output. Three different layers for input, output and hidden neurons has been included in the network. Ten numbers of neurons was used in hidden layer. In the open loop structure, all the training validation and testing is done in the open loop.

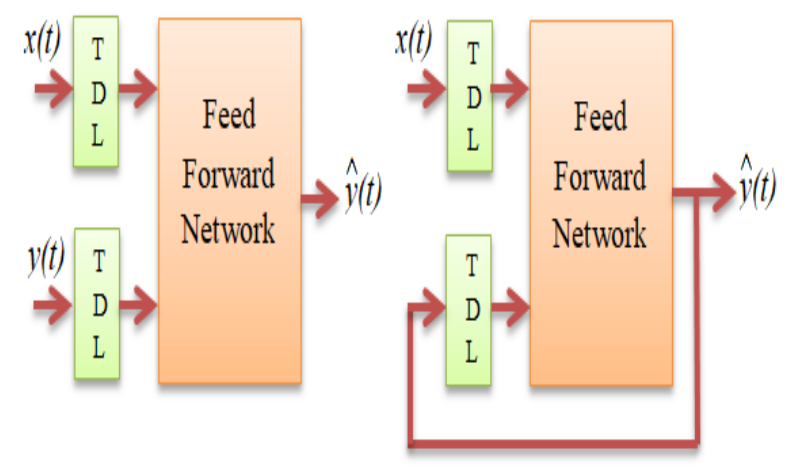

Figure 6.(a) Series Parallel architecture

6.(b)Parallel architecture

Architectural design of the network used is shown below by the "fig.7 (a) and (b)" for open and closed network structure. Close loop architecture is used to execute prediction iteration for next time steps.

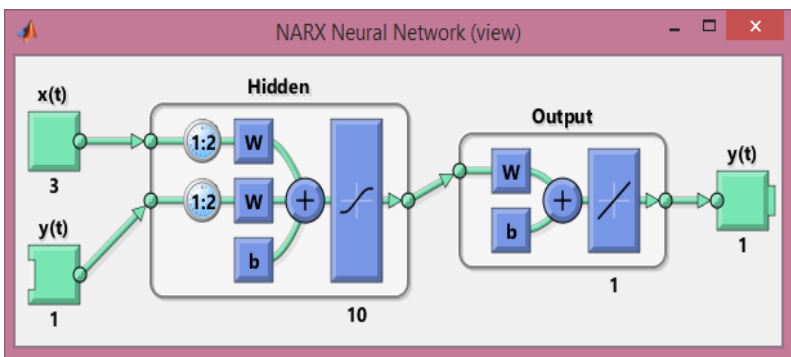

Fig. 7(a). Open loop network structure

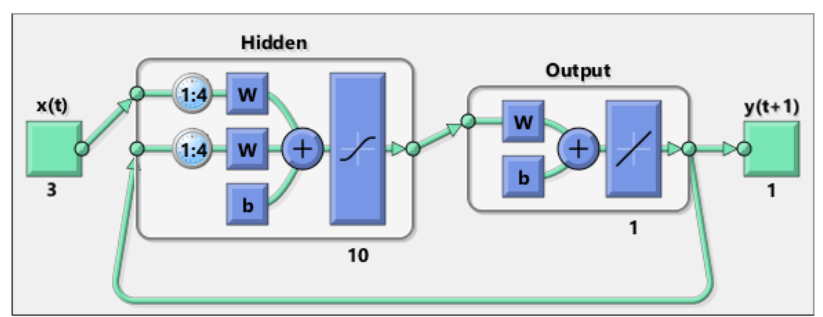

Fig. 7(b). Close loop network structure

The training function used is Scaled Conjugate Gradient (training) because Jacobian training is not supported in GPU programming. Choosing the type of training function is not always easy, as there are many functions available in Neural Network and it depends on the type of problem. Scaled Conjugate Gradient (training) functions do not use a line searching algorithm for looping that will prevent the timeconsuming searching mechanism [23]. For evaluating the performance of the network, both Mean Square Error (MSE) and Correlation Coefficient (R) has been used. The formula for the two is represented in "(3)" and "(4)" below:

$$
\begin{gathered}
\text { MSE }=1 / \mathrm{n} \sum_{i-1}^{\mathrm{n}(y i-\overline{y i})^{2}} . \\
\mathrm{R}=\sqrt{\frac{\sum(\mathrm{yi}-\overline{\mathrm{yi}})^{2}}{\sum_{\mathrm{yi}}^{2}-\frac{\sum \overline{y i}^{2}}{n}}} .
\end{gathered}
$$

, where

$\mathrm{n}=$ Data set number.

yi $=$ Water level observed.

$\bar{y} \mathrm{i}=$ Water level predicted.

For comparing the performance of GPU over CPU and parallel computing, there is a need to know the computational power of the GPU. The amount of memory available for GPU computation and how much data is transferred from CPU to GPU must be analysed. In this paper different strategy like serial computing, parallel computing and GPU computing is compared and then analysis has been done to find out which strategy gives accurate results with high processing speed. 


\section{PLATFORM USED}

The platform used in this paper is MATLAB. MATLAB platform is the most capable system to be used for Deep Learning. It basically works with a matrix that is the main input feature. MATLAB performance is improved by using additional toolboxes. It supports different open-source deep learning structures like ONNX to perform operations. MATLAB is best suited for deep learning architecture due to the following reasons:

1. Preprocess datasets before training.

2. It supports implementation of program with multi programs.

3. Interoperability with different framework.

MATLAB provides many toolboxes, out of which Deep Learning Toolbox is used in this work. This tool executes a plan for constructing and training deep networks. With the help of applications and graph plotting, users can be visualized about the activation and network structure for supervising momentum. For large data set training GPUs are used instead of multicore CPUs or clusters and cloud services can also be used. The training test was carried on the GPU on an Intel computer with specification i5-4790K 1.60GHz, 8GB RAM, equipped with an NVIDIA GPU GeForce MX110, DDR3, GDDR5 of the memory interface, 384 CUDA cores, compliant with Maxwell microarchitecture.

\section{RESULTS AND DISCUSSION}

This section deals with the results related to the training of the network using CPU, Parallel execution and GPU computing."Fig.8 (a)" shows the graphs plotted for checking the training performance graph concerning the Mean Squared Error (MSE) calculated using CPU.

Training performance graphs for parallel and GPU computing are represented by "(b)" and "(c)".It is clear from the below graphs that the performance for validation is best at epoch 102, 35 and 9 for CPU, parallel, and GPU respectively.

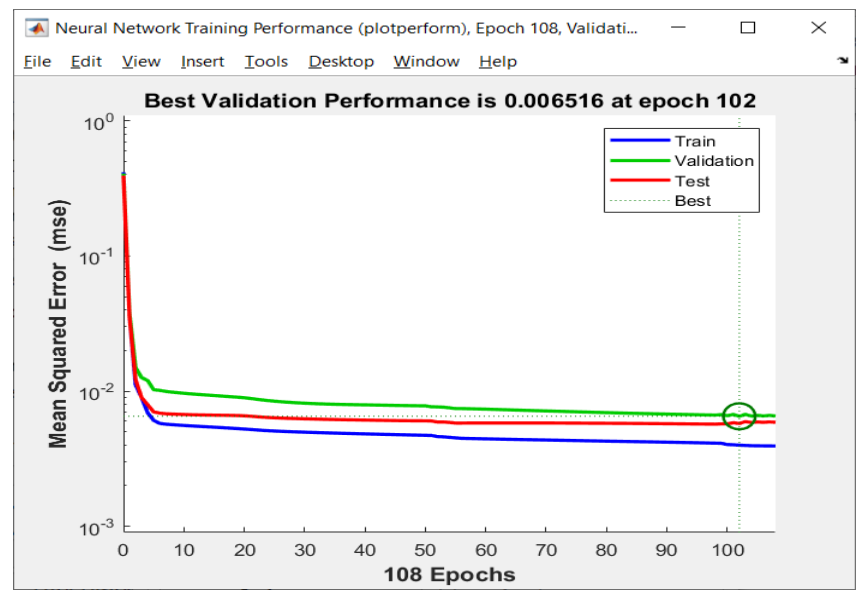

Fig. 8(a) Training performance Graph for CPU

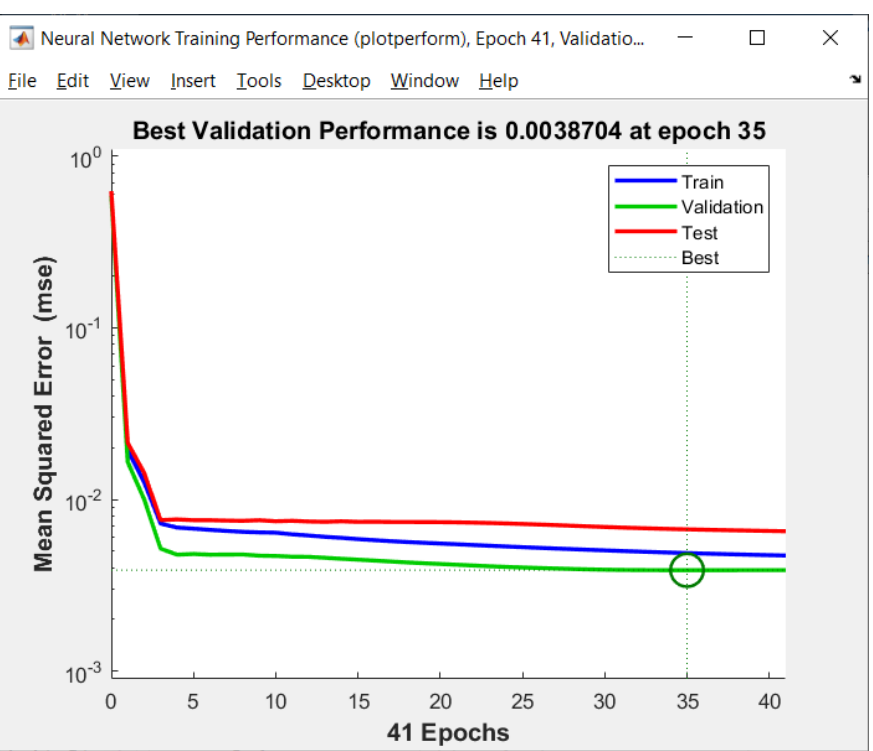

Fig. 8(b) Training performance Graph for Parallel Computing

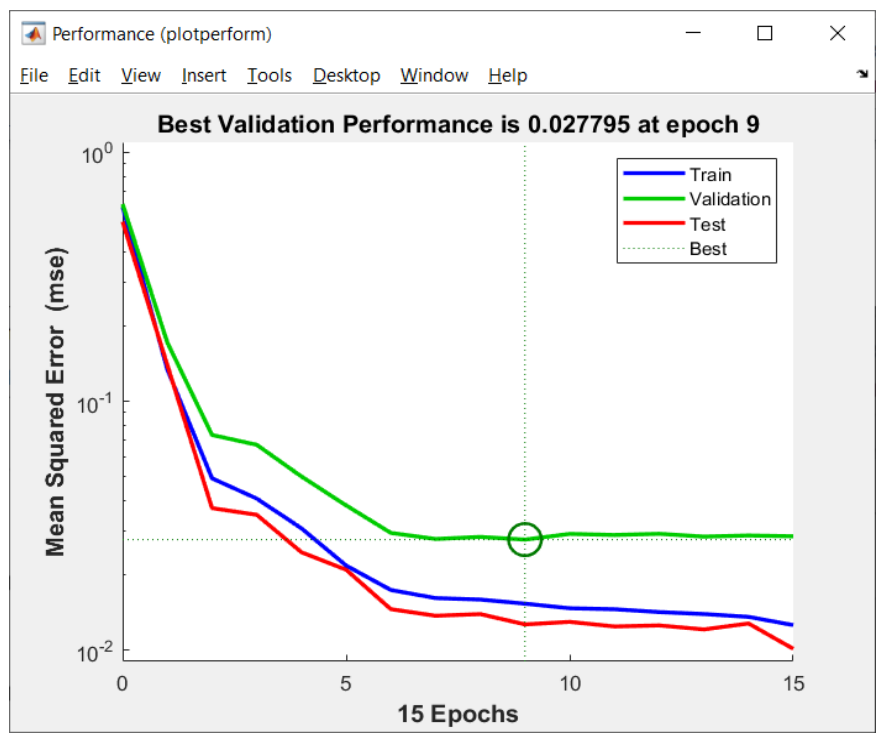

Fig. 8(c). Training performance Graph for GPU

This means that the performance for validation reached a minimum at this epoch and continues to more steps earlier than training stopped. From these graphs, it is clear that performance throughput increases with parallel computing and GPU computing over the CPU.

The Regression plot shows the connnection between the observed output after training the network and the predicted output. The dashed line in the graph shows the perfect target in each graph, which means observed and predicted output is exactly equal,and thick line shows the line of best fit linear regression . $\mathrm{R}$ is the regression value which indicates the type of relationship exists between variables. If its value is equal to 1 , this implies that variables exhibits perfect I"inear relationship ; if its value is 0 , than there is no relationship exhibits between them . In"fig.9" regression plot shows the value of $\mathrm{R} \approx 1$ for training, validating and testing, which means the data indicates a good fit.

Published By:

Blue Eyes Intelligence Engineering \& Sciences Publication

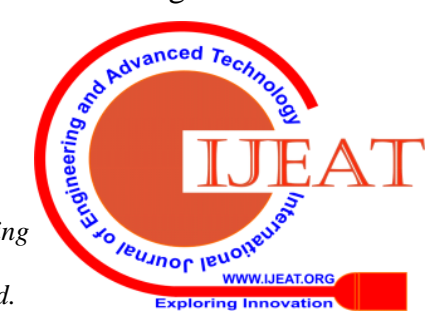




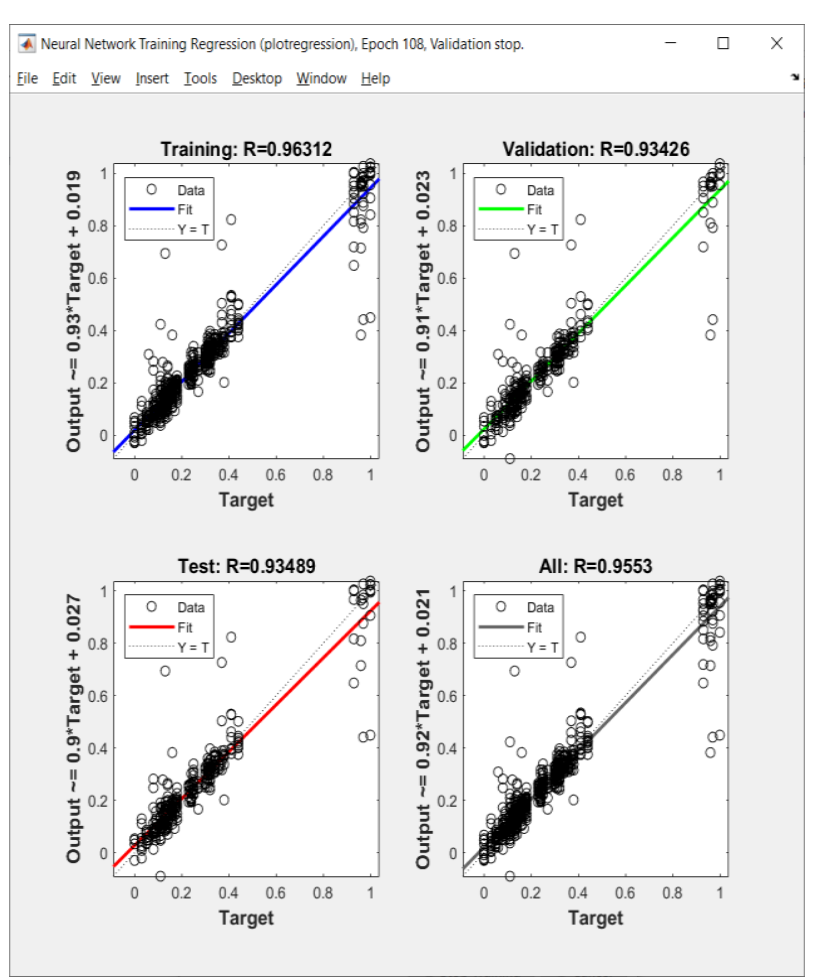

Fig. 9. Regression Plot

Given the table, Table I show the type of technique used for execution with their Regression value, Mean Square Error (MSE) value and Elapsed time for training the network. MSE is the algorithm for supervised learning where the desired output with a given input is also present.MSE explains the closeness of a set of points with a regression line. This is done by calculating the errors and by squaring them. The minimum value of MSE shows the line of good fit. The value of MSE calculated for training the network is the least for GPU that is 0.0039821 . This value represents that the MSE is very small, MSE $\approx 0$, which results in the line of best fit. Whereas the coefficient of regression display the changes in mean for dependent variable where the value of one independent variable makes adjustments and while other independent variable are taken constant. The value of regression varies from 0.9553 to 0.96337 for training, the network through CPU, parallel and GPU approach. Again the value of regression is highest for GPU which represents the good correlation between observed and predicted data sets. The time required by the method for training the Neural Network is called as elapsed time. GPU takes fewer time frames for training the network on same sample size with respect to CPU and parallel execution.

Table I. Computing technique with their elapsed time

\begin{tabular}{|l|l|l|l|}
\hline $\begin{array}{l}\text { Computing } \\
\text { Technique }\end{array}$ & $\begin{array}{l}\text { Mean Square } \\
\text { Error (MSE) }\end{array}$ & Regression & $\begin{array}{l}\text { Elapsed } \\
\text { Time }\end{array}$ \\
\hline CPU Computing & 0.0065159 & 0.9553 & 11.4349 \\
\hline Parallel Computing & 0.0057623 & 0.95169 & 4.6007 \\
\hline GPU Computing & 0.0039821 & 0.96337 & 0.9937 \\
\hline
\end{tabular}

Table II below represents the best performance values for different performance types over CPU, parallel and GPU execution. The performance value for training network is good on GPU for the given dataset, which represents that it can be applied to test the network for further new dataset.

Table II. Performance table

\begin{tabular}{|l|l|l|l|}
\hline Performance Type & CPU & Parallel & GPU \\
\hline Training Performance & 0.0046 & 0.0049 & 0.0024 \\
\hline Validation Performance & 0.0065 & 0.0039 & 0.0051 \\
\hline Testing Performance & 0.0058 & 0.0067 & 0.0033 \\
\hline Closed Loop Performance & 7.0676 & 0.5712 & 0.1201 \\
\hline Multi Step Performance & 0.0020 & 0.1201 & 0.0012 \\
\hline Step Ahead Performance & 0.0206 & 0.0050 & 0.0042 \\
\hline
\end{tabular}

\section{CONCLUSION}

In this paper, a Deep learning approach has been applied to predict the level of water-based on three parameters i.e. temperature, rainfall, and water requirement. Thirteen years of data from 2006 to 2018 of the Faridabad zone has been used to predict the level of water with the help of CPU, Parallel, and GPU computing. From the results it can be concluded that GPU provides faster execution with minimum error rate than other two execution techniques and also improves overall system performance. The overall study of results proved that the Deep Learning approach was best suited for providing the best fit of the curve for predicting the values with GPU computing over CPU and Parallel.

Impact of expanding population, will raised the daily demand of water, which results in exhaustion of ground water level to disquieting phase. From the results it can be easily estimated that the level of water drop off every year approx. 25\%. If the same situation continues than there would be no ground water available for Faridabad zone in future. In "Ref. 19" they concluded that Faridabad comes under the zone of no ground water up to 2050 due to the present situation of ground water in Faridabad district. In this research work the results verified this condition by experimental results.

\section{FUTURE SCOPE}

The use of a Deep Learning approach with GPU for water level prediction is advantageous over other techniques. Despite its successful application, it is a crucial task to choose the best alternative for network architecture, transfer function, frequency of hidden layer neurons and different approach for predicting the network. Here, the Deep Learning model with GPU is compared with CPU and parallel computing for prediction. The present study is restricted to the local architecture as only three input parameters are checked out thoroughly.

\section{Published By:}

Blue Eyes Intelligence Engineering \& Sciences Publication

(C) Copyright: All rights reserved.

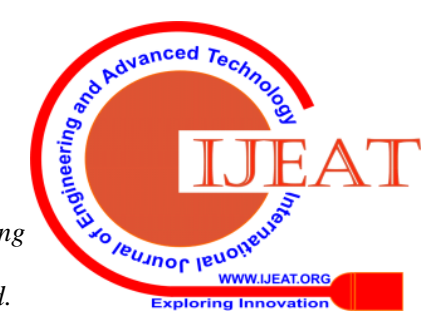


In the future, work can be expanded by adding more parameters to the study and by applying different network architecture, transfer function and number of hidden layers that will be relevant for prediction and will provide better results.According to the current situation, the Faridabad zone will suffer from the deterioration of groundwater in the future as level of water decreases with the increasing population, temperature, low rate rainfall and with the increasing demand for water. For conservation of water need for active participation from people of different sectors like the forest department, pollution control department, a citizens group, media group, and conservation organizations, etc. will be required. There is a need to promote the consciousness of conservation of water through education, guidance, and motivation. Concept of rainwater harvesting, zero discharge industry, water-saving techniques, micro-irrigation should be applied.

\section{ACKNOWLEDGEMENTS}

In performing this research, had to take the help and guideline of some data hail from Water Resources Information of Indian System (WRIS), who deserve our greatest gratitude and thank our colleagues from BITS, Pilani (Rajasthan) who provided insight and expertise that greatly assisted the research.

\section{REFERENCE}

1. Abdul Wahab Qurashi, and Violeta Holmes, "Comparison of Deep Neural Network approach in text and image classification using CPU and GPU systems", Emerging Technology Conference 2019, At University of Huddersfield, 2019, pp.1-5.

2. Rachit Kumar Agrawal, "Difference between Machine Learning, Deep Learning and Artificial Intelligence, https://medium.com/@UdacityINDIA/difference-between-machinelearning-deep-learning-and-artificial-intelligence-e9073d43a4c3.

3. Neeru Singh, and Supriya P.Panda," Enhancing the Proficiency of Artificial Neural Network on Prediction with GPU', IEEE International Conference on Machine Learning, Big Data, Cloud and Parallel Computing (Com-IT-Con) 2019, pp.67-71.

4. Victor W Lee, Changkyu Kim, Jatin Chhugani, Michael Deisher, Daehyun Kim, Anthony D. Nguyen, Nadathur Satish, Mikhail Smelyanskiy, Srinivas Chennupaty, Per Hammarlund, Ronak Singhal, and Pradeep Dubey, "Debunking the 100x GPU vs. CPU myth: an evaluation of throughput computing on CPU and GPU", ACM SIGARCH Computer Architecture News, 2010, vol.38, pp. 451-460. 10.1145/1816038.1816021.

5. Sit Muhammed, and Demir Ibrahim, "Decentralized Flood Forecasting Using Deep Neural Networks”, CoRR. Journal, 2019, EarthArXiv - arXiv: 1902.02308 [cs.LG] - doi:10.31223/osf.io/e9xqr.

6. Yu Wang, Victor Lee, Gu-Yeon Wei, and David Brooks, "Predicting New Workload or CPU Performance by Analyzing Public Datasets", ACM Trans. Archit. Code Optim, 2019, vol.15 (4), Article 53. pp. 53:1--53:21.

7. Matthew Mayo , "Deep Learning Key Terms, Explained", KDnuggets, 2016, https:/www.kdnuggets.com/2016/10/deeplearning-key-terms-explained.html.

8. Meera Narvekar, and Priyanca Fargose, "Article: Daily Weather Forecasting using Artificial Neural Network", International Journal of Computer Applications, 2015, vol. 121(22), pp. 9-13.

9. Ranjan Aneja, "Ground Water Level in Haryana: A challenge for Sustainability", International Journal of Research and Analytical Reviews, vol. 4(3), pp. 43-48.

10. Carl Steidley, Alexey Sadovski, Phillipe Tissot, Ray Bachnak, and Zack Bowles, "Water Level Prediction with Artificial Neural Network Models", 2015.pp. 1-7.

11. Zack Bowles, Philippe E. Tissot, Patrick Michaud, and Alexey Sadovski, “ Artificial Neural Network Predictions of Water Levels in a Gulf of Mexico Shallow Embayment”, Mathematics Magazine:
Theory and Applications, 2005, vol. 12(1 \& 2), pp.139-150, DOI: 10.15517/rmta.v12i1-2.258.

12. Rosmina Bustami, Nabil Bessaih, Charles Bong, and Suhaila Suhaili, "Artificial Neural Network for Precipitation and Water Level Predictions of Bedup River", IAENG International Journal of Computer Science, 2007, vol. 34, pp. 1-7.

13. M.P. Rajurkar, U.C. Kothyari, and U.C. Chaube, "Artificial Neural Networks for daily rainfall- runoff modeling”, Hydrological Sciences Journal, 2002, vol. 47(6), pp.865-877, DOI: 10.1080/02626660209492996.

14. Haytham Assem, Salem Gharbia, Gábor Makrai, Paul Johnston, Laurence Gill, and Francesco Pilla, "Urban Water Flow and Water Level Prediction Based on Deep Learning”, in ECML PKDD 2017: Machine Learning and Knowledge Discovery in Databases, Springer, Cham., , 2017, pp. 317-329.

15. Fatih Unes, Mustafa Demirci, Eyup Ispir, Yunus Ziya Kaya, Mustafa Mamak, and Bestami Tasar, "Estimation of Groundwater level Using Artificial Neural Network: a Case Study of Hatay-Turkey", in Proc 10th Int. Conf. Environmental Engineering, 2017 pp. 1-6.

16. Pallavi porte, Rajendra Kumar Isaac, KipooKiran Singh Mahilang, Khilendra Sonboier, and Pankaj Minj, "Groundwater Level Prediction using Artificial Neural Network Model”, International Journal of Current Microbiology and Applied Sciences,2018, vol. 7(2), pp. 2947-2954. DOI: 10.20546/ijcmas.2018.702.358.

17. Igor Coelho, Vitor Coelho, Eduardo Luz, Luiz Ochi, Frederico Guimaraes, and Eyder Rios, “ A GPU deep learning metaheuristic based model for time series forecasting”, Applied Energy, 2017, vol.201, pp. 412-418.

18. Viacheslav Khomenko, Oleg Shyshkov, Olga Radyvonenko, and Kostiantyn Bokhan, "Accelerating recurrent neural network training using sequence bucketing and multi-GPU data parallelization", CoRR. Journal, 2017, vol. abs/1708.05604, EarthArXiv arxiv.org/abs/1708.05604.

19. Thamar Abdul Rahman, SherringArpan, and Ahsan MD Jafri, "Ground Water Modeling of Faridabad District, Haryana, India", International Journal of Agriculture sciences, 2016, vol. 8(47)., pp. 1957-1965.

20. L. Shaikh, and K. Sawlani, "A Rainfall Prediction Model Using Artificial Neural Network, International Journal of Scientific Research in Network Security and Communication, 2017, vol. 5 (1), pp. 24-28.

21. Bhavna Sharma, and K. Venugopalan, "Comparison of Neural Network Training Functions for Hematoma Classification in Brain CT Images”, IOSR Journal of Computer Engineering, 2014, vol.16, pp. 31-35. 10.9790/0661-16123135.

22. Annalisa Di Piazza, Maria Carmela Di Piazza, and Gianpaolo Vitale, "Solar and wind forecasting by NARX neural networks, Renew. Energy Environ. Sustain, 2016, vol. 1 (39), pp.1-5.

23. Al-Sbou, Yazeed A., and Khaled Mohammad Alawasa, "Nonlinear Autoregressive Recurrent Neural Network Model for Solar Radiation Prediction, International Journal of Applied Engineering Research, 2017, vol. 12 (14), pp. 4518-4527.

\section{AUTHORS PROFILE}

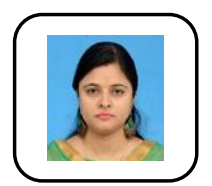

Neeru Singh holds an M.tech degree in computer science and engineering from Maharishi Dayanand University, Rohtak, India. She is pursuing a Ph.D. degree in computer science and engineering from Manav Rachna International Institutes of Research and Studies (MRIIRS), Deemed to be university, Faridabad, India. She is currently working as an assistant professor in computer application department, Rawal Institute of Management, Faridabad, India and has 3 years of experience in teaching. Her major field of study is artificial neural network. 
Supriya P. Panda received her Ph.D. degree in formal languages and automata theory from Bowling Green State University, Ohio, USA in 1990.Prof. Panda received best teaching fellow at Bowling Green State University, Ohio, USA during her MS and Ph.D. in 1985-1990. She has over 30 years of experience in teaching, mentoring, guiding as a researcher to B.tech and M.tech students. At present she is the Head of the Department (HOD) of Faculty of engineering in computer science at Manav Rachna International Institutes of Research and Studies (MRIIRS), Faridabad, India. 